\title{
Quantification of the leucocyte influx into rabbit ileal loops induced by strains of Salmonella typhimurium of different virulence
}

\author{
T. S. WALLIS, R. J. H. HAWKER*, D. C. A. CANDYt, G.-M. OI\$, G. J. CLARKE, \\ K. J. WORTON, M. P. OSBORNE
}

Departments of Microbiology, *Surgery and $\ddagger$ Physiology, University of Birmingham, PO Box 363, Birmingham B15 2TT, and †Institute of Child Health, Francis Road, Birmingham B16 $8 E T$

\begin{abstract}
Summary. Leucocyte influx into rabbit ileal loops, induced by strains of Salmonella typhimurium of different virulence, was assessed with ${ }^{111}$ Indium-labelled leucocytes. Strains fell into two groups on the basis of their leucotactic potential: "virulent" strains (which induced fluid secretion) caused a dose-dependent leucocyte influx; strains which did not induce fluid secretion failed to induce a significant leucocyte influx. Fluid secretion was never observed in the absence of leucocyte influx, but leucocyte influx per se did not induce fluid secretion. The phenotype of the challenge inoculum influenced fluid secretion; young log-phase organisms induced fluid secretion with a higher frequency than overnight cultures. These findings support earlier evidence implicating leucocytes in an interactive but not exclusive role in the genesis of salmonella-induced fluid secretion. They suggest, though do not prove, that interaction of leucocytes with the appropriate phenotype of organisms results in the release of a host-derived or bacterial secretagogue, or both. The bacterial factor may or may not be the antigen related to cholera toxin, described previously.
\end{abstract}

\section{Introduction}

The mechanisms involved in the induction of fluid secretion by salmonellae are poorly understood, and have been reviewed by Stephen et al. (1985). Previously we have shown that "virulence" of Salmonella typhimurium - ability to induce fluid secretion in rabbit ileal loops (Wallis et al., 1986a; Clarke et al., 1988)—does not correlate with ability to make enterotoxin in vitro (Wallis et al., 1986a) or ability simply to adhere to intestinal tissue (Worton et al., 1989).

Subjective histological evidence for the possible involvement of leucocytes in the genesis of fluid secretion was obtained by Giannella et al. (1973), who examined various strains of $S$. typhimurium in the rabbit ileal loop test (RILT) - a good model for $S$. typhimurium diarrhoeal disease (Stephen et al., 1985). Giannella and co-workers found that strains TML, W1 18 and M206 invaded rabbit ileal mucosa

Received 7 Oct. 1988; accepted 21 April 1989.

$\S$ Present address: Institute of Epidemiology and Microbiology, PO Box 5, Changping, Beijing, China.

Correspondence should be sent to Dr J. Stephen, Department of Microbiology, University of Birmingham, PO Box 363, Birmingham B15 2TT. and induced fluid secretion; such strains were designated biotype $(++)$ by Clarke et al. (1988). Strains SL1027 and LT7 invaded the ileal mucosa but induced negligible fluid secretion-biotype (+ $-)$; strain Thax-1 neither invaded the mucosa nor induced fluid secretion-biotype $(--)$. Examination of histological sections suggested that strains TML and W118 induced an intense, but unquantified, inflammatory cell influx into infected intestinal mucosa, whereas strain Thax-1 did not evoke such influx; strains M206, SL1027, and LT7 evoked an intermediate inflammatory reaction (Giannella et al., 1973). We have re-examined these strains in the RILT and, with the exception of strain M206, which in our hands is of biotype $(+-)$, they have retained their biological properties (Wallis et al., 1986a). Giannella (1979) demonstrated the negative corollary to the data on inflammatory cell influx : in rabbits rendered neutropaenic with nitrogen mustard, salmonella-induced fluid secretion in RILs was inhibited.

Additional indirect evidence, possibly implicating polymorphonuclear leucocytes in the genesis of fluid secretion, stems from the use of indomethacin which inhibits the synthesis of prostaglandins. The latter are generated by several cell types, including 
neutrophils, during the inflammatory process (Higgs et al., 1975) and have been demonstrated to induce fluid secretion in the intestinal mucosa (Pierce et al., 1971; Matuchansky and Bernier, 1973). Two types of experiment have been performed previously.

First, the fluid response to challenge with live organisms was studied in RILs and monkeys treated with indomethacin. Gots et al. (1974) showed that if rabbits were pre-treated with indomethacin, fluid secretion in ileal loops challenged with $S$. typhimurium was almost completely inhibited, whereas bacterial invasion and the accompanying inflammatory cell influx appeared normal. Fluid secretions evoked by Vibrio cholerae, Shigella flexneri and cholera toxin (CT) were also reduced by $50 \%$. Giannella et al. (1977) also showed that salmonellainduced diarrhoea in orally inoculated monkeys could be inhibited by prior treatment with indomethacin.

Second, Giannella et al. (1975) examined also the levels of adenylate cyclase in RILs challenged with CT and with $S$. typhimurium strains TML $(++)$ and SL1027 (+ -). CT and strain TML caused increase in adenylate cyclase activity and also fluid secretion, whereas strain SL1027 did not. Indomethacin pre-treatment inhibited the increase in adenylate cyclase activity and fluid response in RILs challenged with strain TML. In the case of $\mathrm{CT}$, adenylate cyclase activity was not reduced but the fluid response was reduced by $50 \%$. Giannella et al. (1975) proposed that the previously reported failure of strain SL1027 (+ - ) to induce more than a mild inflammatory cell influx (Giannella et al., 1973) was the reason for its failure to induce fluid secretion. These data question the exclusivity of the role played by adenylate cyclase, and hence cyclic nucleotides, in both CT- and $S$. typhimuriuminduced fluid secretion. They suggest a role for an indomethacin-sensitive (possibly prostaglandin) mediator, though one cannot assume that the indomethacin acted only upon prostaglandin synthesis.

In contrast to the above, Duebbert and Peterson (1985) denied a role for inflammatory cells in the induction of fluid secretion, and refocussed attention on the possible role of a CT-like salmonellaenterotoxin acting via cyclic adenosine monophosphate (cAMP) and prostaglandins originating from epithelial cells. However, first, their data (in their table 1) give no evidence that cAMP or prostaglandins are involved in the induction of fluid in RILs challenged with $S$. typhimurium; there were no significant differences in the levels of either cAMP or prostaglandins produced by fluid-inducing and non-fluid-inducing strains. Moreover, the degrees to which these levels were depressed by indomethacin were similar for both fluid-inducing and nonfluid-inducing strains. Second, we have shown that $(+-)$ and $(++)$ strains produce enterotoxin(s) in vitro equally well; and we have argued that enterotoxigenicity, at least as determined in vitro, cannot per se be the sole factor which determines fluid secretion by $S$. typhimurium in vivo (Wallis et al., 1986a).

In this laboratory, we have attempted to resolve this controversy. In a previous study, with the same strains as those used by Giannella's and Peterson's groups, we observed consistently that the onset of fluid secretion in RILs coincided with an inflammatory cell influx into the ileal mucosa (Wallis et $a l ., 1986 b$ ). In the present paper we describe experiments to quantify the influx of leucocytes into RILs challenged with strains of different virulence, in an attempt to strengthen or weaken the possible correlation between fluid secretion and a role for leucocytes. The approach is based on techniques used by clinicians to locate internal abscesses by use of radio-labelled leucocytes (Coleman et al., 1980).

\section{Materials and methods}

\section{Bacterial strains}

Six strains of $S$. typhimurium (TML, W118, LT7, SL1027, M206 and Thax-1) have been used previously (Wallis et al., 1986a; Clarke et al., 1988), and their biotypes are stated in the Introduction. Strain WAKE was isolated from a patient in an outbreak of $S$. typhimurium food poisoning at Stanley Royd Hospital, Wakefield, and was kindly donated by Leeds Regional Public Health Laboratory; it was of biotype $(++)$ in the RILT. Strains were stored at $-70^{\circ} \mathrm{C}$ in equal proportions of glycerol and Muller-Hinton broth.

\section{Rabbits}

New Zealand White rabbits $(2 \cdot 5-3 \mathrm{Kg})$ were obtained from Regal Rabbits, Great Bookham, Surrey, and from Ranch Rabbits, Crawley Down, Sussex. All were bled on arrival, and their sera screened for antibodies to $S$. typhimurium by slide agglutination, and to $\mathrm{CT}$ by immunoblotting (Clarke et al., 1988).

\section{Preparation of inocula}

Inoculum $A$, as used initially, was prepared as follows. Strains were streaked on MacConkey's agar and incubated at $37^{\circ} \mathrm{C}$ overnight. A small portion of each of $c .15$ colonies was suspended in $10 \mathrm{ml}$ of sterile saline, and 
$10 \mathrm{ml}$ of Hartley Digest Broth (HDB; Oxoid) were inoculated with $25 \mu$ lof this suspension. After incubation at $37^{\circ} \mathrm{C}$ overnight, organisms were washed in sterile isotonic saline $(\mathrm{NaCl} 9 \mathrm{~g} / \mathrm{L}$ in distilled water), and resuspended in fresh $\mathrm{HDB}$; then $c .1 \times 10^{9}$ viable bacteria were inoculated into RILs which were constructed and challenged as described previously (Wallis et al., 1986a).

Inoculum $B$. The above protocol was modified midway through this work-see Qi et al. (1989) and Results and Discussion. HDB was inoculated heavily from $c$. five colonies on a MacConkey-agar plate, and incubated for only $4.5 \mathrm{~h}$. Again, c. $1 \times 10^{9}$ viable washed bacteria, suspended in fresh $\mathrm{HDB}$, were used to challenge the RILs.

Positive control loops were inoculated with $1 \mu \mathrm{g}$ of CT in $1 \mathrm{ml}$ of sterile saline.

Negative control loops were inoculated with $1.5 \mathrm{ml}$ of sterile HDB. Results were considered valid only if negative control loops were flat and CT loops had V/L (volume $(\mathrm{ml}) /$ length $(\mathrm{cm})$ of loop) ratios $>0.5$; usually the $\mathrm{V} / \mathrm{L}$ ratios of CT loops were in the range $1.5-2.5 \mathrm{ml} /$ $\mathrm{cm}$.

\section{Immunofluorescence microscopy}

Full thickness mucosal biopsies were sampled from ileal loops and were fixed, sectioned and stained by an indirect fluorescent-antibody method to detect salmonellae as previously described (Wallis et al., 1986b).

\section{Quantification and imaging of the inflammatory cell influx}

The white-cell labelling method was a modification of that used by Hawker et al. (1985)-see fig. 1. The experimental protocol was designed such that the labelled leucocytes were re-injected just before the expected leucocyte influx into the intestinal mucosa, which occurred reproducibly in this experimental model (Wallis et al., 1986b).

Four hours after challenge of the loops, $17 \mathrm{ml}$ of blood were taken from an ear vein of each animal and added to $3 \mathrm{ml}$ of acid citrate $\left(\mathrm{Na}_{3}\right.$ citrate $25 \mathrm{~g} / \mathrm{L}$, citric acid, anhydrous, $13.6 \mathrm{~g} / \mathrm{L} ; \mathrm{pH} 4.9)$ in a polystyrene universal container (Sterilin 128/B); $5 \mathrm{ml}$ of dextran (mol. wt $c$. $500000) 6 \% \mathrm{w} / \mathrm{v}$ in isotonic saline were then added to the blood, which was gently mixed and allowed to stand at $37^{\circ} \mathrm{C}$ for up to $1 \mathrm{~h}$ to permit erythrocyte sedimentation. Sedimentation was allowed to occur sufficiently to yield c. $10 \mathrm{ml}$ of erythrocyte-free supernate which contained plasma, leucocytes and platelets; and $8 \mathrm{ml}$ of this supernate was transferred to a round-bottomed, polystyrene tube (Sterilin, 142AS) with a plastic pasteur

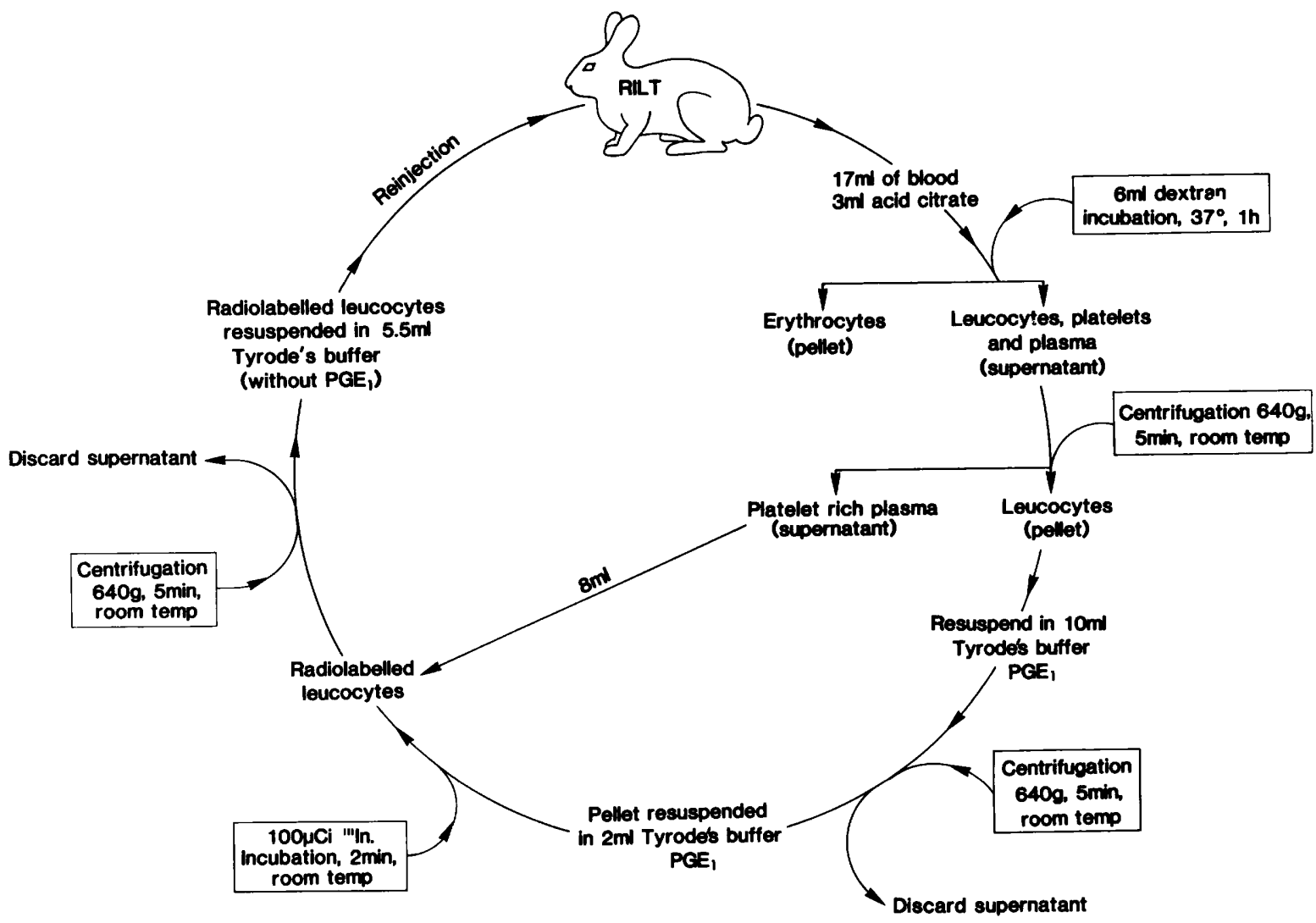

Fig. 1. Schematic diagram for the leucocyte labelling protocol. 
pipette (Appelton Woods, Birmingham). White cells were sedimented by centrifugation $(640 \mathrm{~g}, 5 \mathrm{~min}$ at room temperature); the supernate, containing plasma and platelets, was collected and held at room temperature for use as described below. The pellet, comprising mixed leucocytes and platelets, was suspended in $10 \mathrm{ml}$ of $\mathrm{Ca}^{2+}$ -free Tyrode's buffer $\left(\mathrm{NaCl} 8 \mathrm{~g} / \mathrm{L}, \mathrm{KCl} 2 \mathrm{~g} / \mathrm{L}, \mathrm{NaHCO}_{3} 1 \cdot 0\right.$ $\mathrm{g} / \mathrm{L}, \mathrm{NaH}_{2} \mathrm{PO}_{4} \cdot 2 \mathrm{H}_{2} \mathrm{O} 0.05 \mathrm{~g} / \mathrm{L}, \mathrm{MgCl}_{2} \cdot 6 \mathrm{H}_{2} \mathrm{O} 0 \cdot 4 \mathrm{~g} / \mathrm{L}, \mathrm{D}-$ glucose $1.0 \mathrm{~g} / \mathrm{L} ; \mathrm{pH} 6.5$ ) containing prostaglandin $\mathrm{E}_{1}$ (Sigma) $0.4 \mu \mathrm{g} / \mathrm{ml}$, the latter being included to inhibit platelet aggregation. The leucocytes were recentrifuged $(640 \mathrm{~g}, 5 \mathrm{~min}$, room temperature) and resuspended in $2 \mathrm{ml}$ of $\mathrm{Ca}^{2+}$-free Tyrode's buffer containing prostaglan$\operatorname{din} \mathrm{E}_{1}$.

${ }^{111}$ Indium oxine (Amersham International, Bucks) $100 \mu \mathrm{Ci}$ was added to the leucocyte suspension and incubated for $2 \mathrm{~min}$ at room temperature $; 8 \mathrm{ml}$ of plateletrich plasma were then added to the leucocyte suspension to absorb any unbound isotope; and the leucocytes were recentrifuged ( $640 \mathrm{~g}, 5 \mathrm{~min}$, room temperature). Finally, leucocytes were resuspended in $5.5 \mathrm{ml}$ of $\mathrm{Ca}^{2+}$-free Tyrode's buffer (without prostaglandin $\mathrm{E}_{1}$ ) and reinjected into the test rabbit through a $21 \mathrm{G}$ hypodermic needle via an ear vein; experiments showed that the final suspension contained less than $15 \mathrm{ng}$ of prostaglandin $\mathrm{E}_{1}$. The procedure from bleeding to reinjection took $c .2 \mathrm{~h}$.

Animals were sedated by intravenous injection of pentobarbitone $30 \mathrm{mg} / \mathrm{kg}$ (Willington Medical, Shrewsbury), $12 \mathrm{~h}$ after initial challenge with organisms, and a gamma-scan of the animals was performed with a Gamma-camera-Searle LFOV Camera fitted with a medium energy collimator and information stored on an on-line DEC (PDP) computer. The rabbits were then killed by intravenous pentobarbitone overdose, and the V/L ratios were determined (Wallis et al., 1986a). Ileal loops were excised and trimmed of mesentery and sutures. Mucosa and loop-contents were then counted separately in a $\mathrm{NaI}$ well crystal attached to a rate meter (Nuclear Enterprises, Reading, Berks) set to detect the two photopeaks of ${ }^{111}$ In at 173 and $247 \mathrm{keV}$. The leucocyte influx into the test loops was measured as a ratio of cpm for test loop to cpm for negative control loop in the same rabbit (leucocyte influx ratio).

\section{Results}

\section{Screening of rabbit sera}

Of the rabbits used in this study, $50 \%$ had agglutinating antibodies to $S$. typhimurium. In contrast to those used by Clarke et al. (1988), none of our rabbits had circulating antibodies to CT. There was no correlation between fluid secretion in response to $(++)$ strains, or to $C T$, and the presence of antibodies to $S$. typhimurium.

\section{Rabbit imaging}

Fig. 2 shows a typical gamma-camera ventral image of a rabbit with ${ }^{111}$ In-labelled leucocytes and

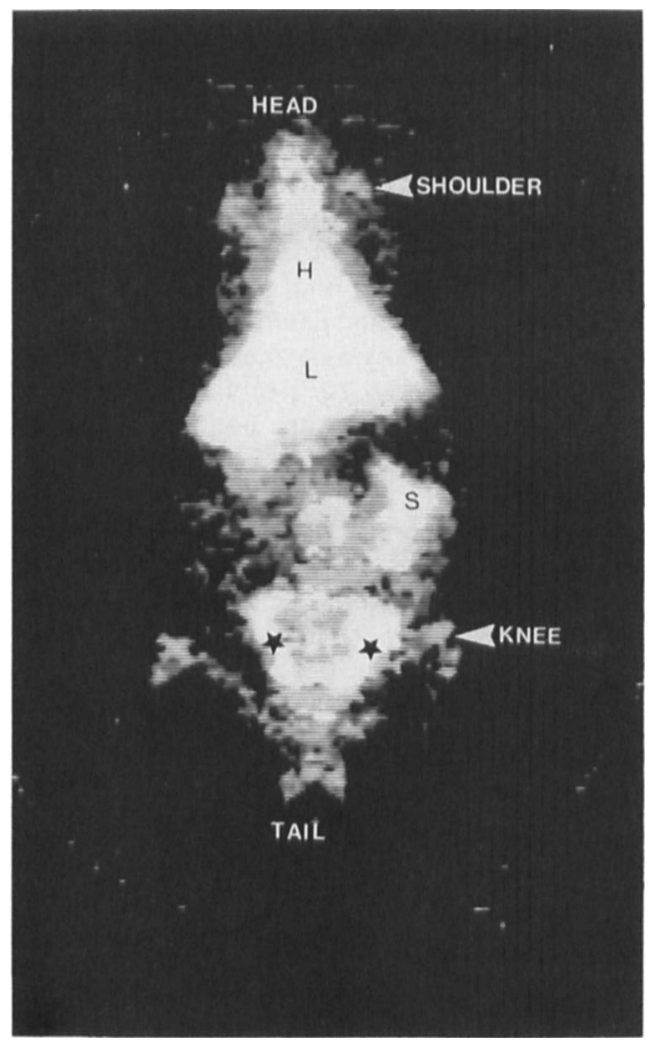

Fig. 2. A typical gamma-camera ventral image of a rabbit with ${ }^{111}$ Indium-labelled leucocytes, $12 \mathrm{~h}$ after ileal loop challenge. Two test loops (*) challenged with $S$. typhimurium strain TML can be seen in the lower abdominal region. The heart $(\mathrm{H})$, liver (L) and spleen (S) can also be seen, as well as a fainter skeletal image (shoulder and knee).

S. typhimurium-infected ileal loops. Light areas representing regions of high ${ }^{111}$ In activity can be seen in the liver, spleen and lower abdomen. The areas of high activity in the lower abdomen represent the two ligated ileal loops infected with strain TML.

\section{Bacterial invasion as assessed by immunofluorescence microscopy}

Subjective examination of mucosa challenged with $(++)$ strains, and sampled at $12 \mathrm{~h}$, revealed many luminal as well as intracellular salmonellae. Though villi were not all equally invaded, the majority were heavily invaded with up to 50 fluorescently stained bacterial foci seen in sections; a few villi showed few or no invading bacteria.

Although fluid secretion did not occur in all loops that were inoculated with $(++)$ strains, there was no obvious difference in the degrees of invasion by 
these strains, between loops which had fluid secretion and those which did not.

\section{Quantification of leucocyte influx induced by CT and} by different biotypes of $S$. typhimurium

In fig. 3, the data have been compiled from 126 loops constructed in 57 rabbits inoculated with either type A (overnight) or type B (log phase) inocula and observed after $12 \mathrm{~h}$. Strains TML, W118 and WAKE were more leucotactic than strains LT7, SL1027, M206 and Thax-1. Only 48\% of the rabbits responded by secreting fluid (V/ $\mathrm{L}=0.5-3.2 \mathrm{ml} / \mathrm{cm}$ ) into RILs challenged with strains TML, W118 and WAKE, of biotype $(++$ )-see below; but the $(+-)$ strains gave consistently negative results $(\mathrm{V} / \mathrm{L}<0.5 \mathrm{ml} / \mathrm{cm})$. In loops challenged with $\mathrm{CT}$, in which an intense fluid response was observed $(\mathrm{V} / \mathrm{L}=1 \cdot 5-2 \cdot 7 \mathrm{ml} / \mathrm{cm})$, the mean leucocyte influx ratio was $2 \cdot 4$.

Because, in the initial experiments with inoculum A, the varied fluid response did not correlate with the serological status of the animals, two changes were made in an attempt to increase the proportion of animals giving the expected fluid response to (+ $+)$ strains. First, animals were left for $24 \mathrm{~h}$ before examination; but this did not increase the proportion of loops with a positive fluid response, though

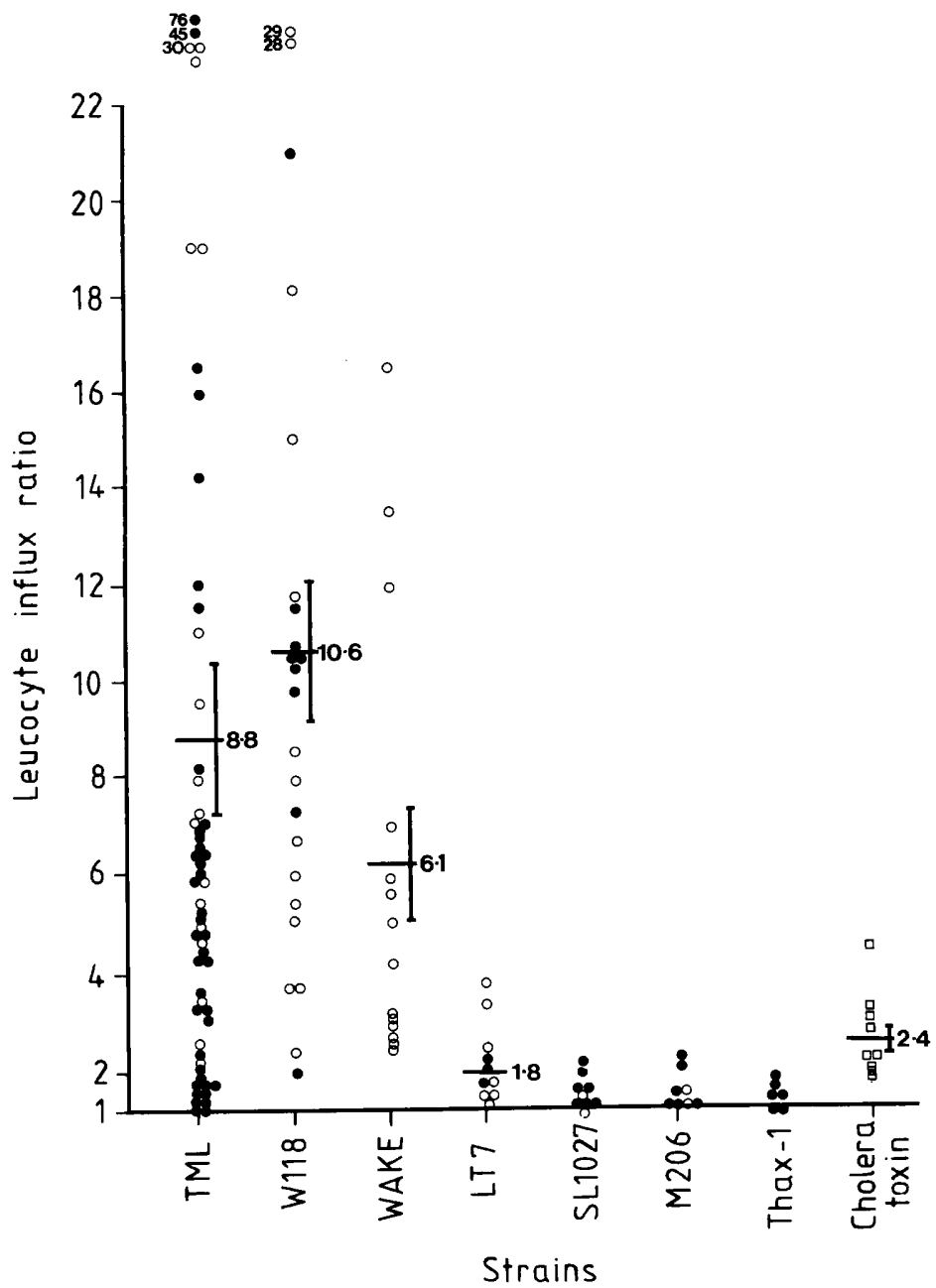

Fig. 3. Leucocyte influxes into RILs induced by strains of $S$. typhimurium of different virulence and by cholera toxin, $12 \mathrm{~h}$ after loop challenge: $=$ loop challenged with inoculum A (overnight growth); $O=$ loop challenged with inoculum B (early log-phase). The mean leucocyte influx and standard error of the mean are indicated for strains TML, W118, WAKE and LT7, and cholera toxin. The data for strains SL1027, M206 and Thax-1 were tightly grouped and therefore the means are not indicated.

Leucocyte influx ratio $=\frac{\mathrm{cpm} \text { for loop challenged with bacteria (or toxin) }}{\mathrm{cpm} \text { for negative control loop }}$. 
it did increase the leucocyte influx into loops challenged with $(++)$ strains and CT (fig. 4). Second, animals were challenged with young logphase organisms (inoculum B) and examined at $12 \mathrm{~h}$ : there was a marked increase in the number of loops showing fluid secretion. This is most clearly shown by re-analysis of the data in fig. 3 , as presented in figs. $5 \mathrm{a}$ and $5 \mathrm{~b}$ showing the leucocyte influxes in loops in which fluid did or did not accumulate. For strains TML and W118 combined, $73 \%$ of all the responders were challenged with inoculum B; only $10 \%$ of the non-responders were challenged with inoculum $B$. The mean leucocyte

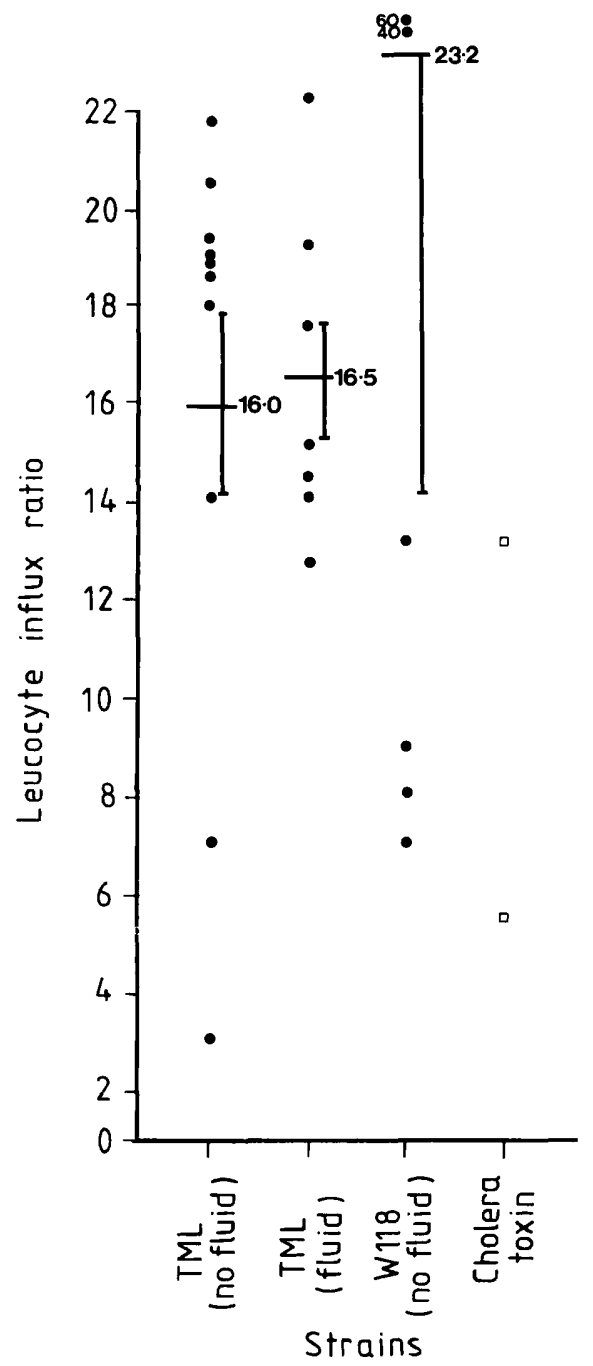

Fig. 4. Leucocyte influxes into RILs (with and without fluid accumulation), $24 \mathrm{~h}$ after loop challenge. Loops were challenged with inoculum A (overnight growth) of $S$. typhimurium strains TML and W118 and with cholera toxin. The mean leucocyte influx (see fig. 3) and SEM are indicated for each strain. (a)

(b)

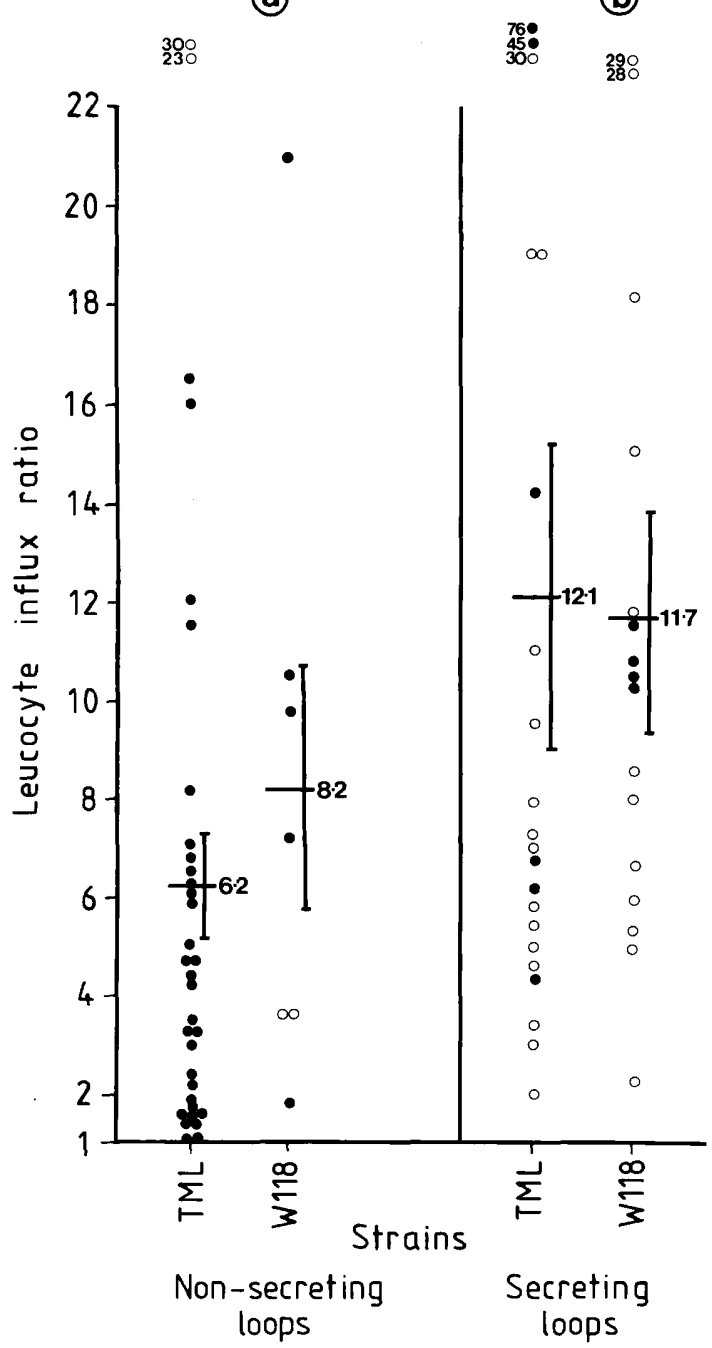

Fig. 5. Leucocyte influxes (see fig. 3) into ileal loops $12 \mathrm{~h}$ after loop challenge. Re-analysis of data in fig. 3. Loops were challenged with $S$. typhimurium strains TML and W118: $O=$ loop challenged with inoculum A (overnight); $O=$ loop challenged with inoculum B (log-phase); a, loops without fluid accumulation; b, loops with fluid accumulation. The mean leucocyte influx and SEM are indicated for each strain.

influx with secreting loops was marginally greater than for non-secreting loops.

The relationship between size of inoculum $B$ and leucocyte influx is shown in fig. 6. There was variation between individual rabbits (in rabbit 4, the leucocyte influx was less intense compared with rabbits 1, 2 and 3), but for each rabbit there was a correlation between leucocyte influx and inoculum size. In addition, with one exception, fluid secretion was observed only in loops challenged with inocula $\geqslant 10^{7}$ organisms. 


\section{Discussion}

We report studies on the host response in experimental $S$. typhimurium infection of ligated RILs, with strains characterised previously and a recent clinical isolate, and a novel application of a technique used routinely in clinical medicine. To our knowledge, this is the first time that virulence of $S$. typhimurium - at least as demonstrated by fluid secretion in RILs- has been correlated with a quantifiable parameter. We found two groups of strains: TML, W118 and WAKE were potent inducers of a leucocyte influx; LT7, SL1027, M206 and Thax-1 did not induce a leucocyte influx significantly above background. These findings thus support the possibility that white cells play a role in the causation of fluid secretion, because only strains TML, W118 and WAKE caused significant fluid secretion in the RIL.

It is clear, however, that a leucocyte influx per se does not induce fluid secretion, since in a large number of experiments (figs. 4 and 5a) high influxes of leucocytes occurred with no associated fluid secretion. This failure to secrete could not be correlated with the presence of anti-salmonella antibodies in the sera of the animals.

By comparison with the $(++)$ strains, CT induced only a slight influx of leucocytes at $12 \mathrm{~h}$, while inducing an intense fluid response. An increased leucocyte influx was seen at $24 \mathrm{~h}$, probably in response to mucosal damage resulting from elevated hydrostatic pressure developing within such ileal loops over the longer period.

Three points emerge which might throw some light on the mechanism(s) of fluid secretion. First, despite the fact that leucocyte influx per se is not directly responsible for salmonella-induced fluid secretion, the latter was never observed in the absence of a significant leucocyte influx. Second, the phenotype of the fluid-inducing strains is obviously important-young cultures induced secretion with a strikingly higher frequency than older cultures (figs. 5a and b). Third, the size of the initial inoculum is important because, with one exception, loop dilation occurred only when the inoculum was $\geqslant 10^{7} \mathrm{cfu} /$ challenge (fig. 6).

We therefore postulate that leucocyte influx into infected intestinal mucosa plays an important part in the genesis of fluid secretion. Interaction of leucocytes with bacteria of the correct phenotype results in the release of a host-derived secretagogue (possibly prostaglandins of neutrophil or other cell origin) or of a bacterial enterotoxin, or both. The data do not allow discrimination between these two possible mechanisms, involving or not involving

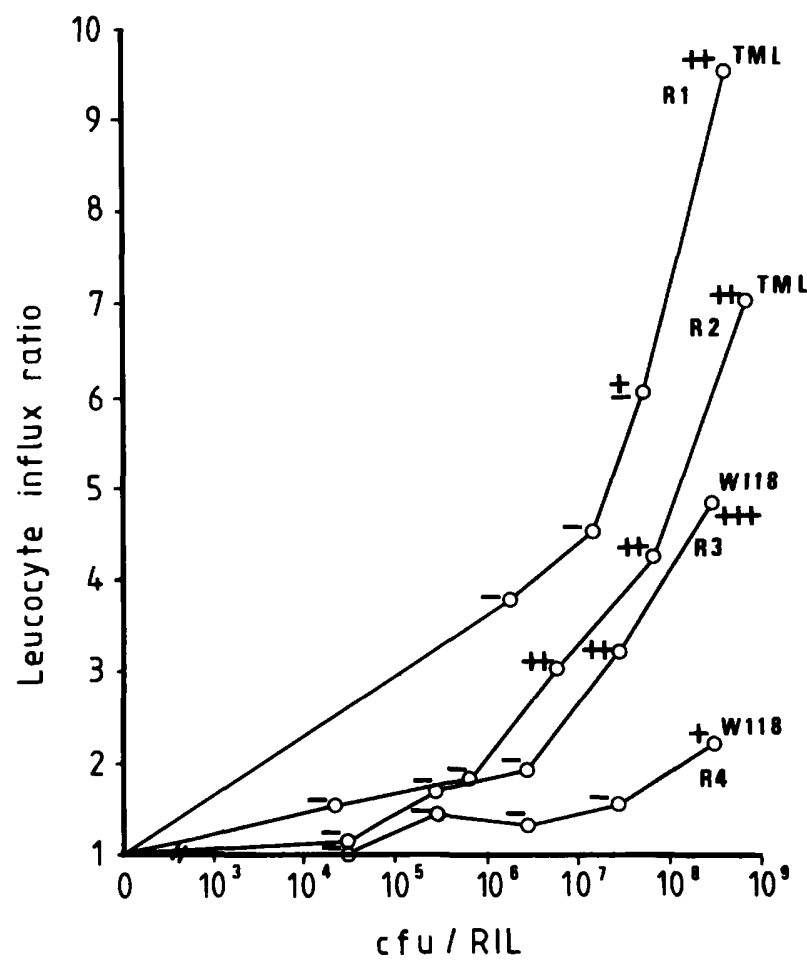

Fig. 6. Leucocyte influxes (see fig. 3) into rabbit ileal loops in four rabbits (R1-R4): dose response induced by inoculum $B$ (log-phase) of $(++)$ biotype of $S$. typhimurium (strains TML and $\mathrm{W} 118)$. Volume/length $(\mathrm{V} / \mathrm{L})$ ratios: $-=<0 \cdot 3 ; \pm=0 \cdot 3-$ $0.4 ;+=0.5-0.9 ;++=1.0-1.5 ;+++=>1.5 \mathrm{ml} / \mathrm{cm}$.

release of prostaglandins; nor need they be mutually exclusive. Whichever is the case, there is clearly an optimal bacterial phenotype since young log-phase cultures are more efficient elicitors of fluid secretion than organisms taken in late-log or stationary phase. We have not yet proved the existence of a CT-like enterotoxin, as demonstrated by Finkelstein et al. (1983); but already, in the strains used in this study, we have shown the existence of a salmonella enterotoxin (Wallis et al., 1986a), and a CT-related antigen (Clarke et al., 1988) which may or may not be a CT-related toxin. The expression of this antigen is dependent on the culture medium and the time at which the culture is examined (Clarke et al., 1988; Qi et al., 1989). There is a higher proportion of organisms bearing this antigen in early log-phase than at subsequent stages of growth. In retrospect, we now see that the biotyping described by Wallis et al. (1986a), in which there was a high degree of reproducibility, was done with inocula of early log-phase organisms. The change to overnight cultures was a logistical convenience, which at first did not appear to affect adversely the 
ability of $(++)$ strains to elicit fluid secretion. Clearly the initial success with older cultures was fortuitous, because subsequently this was not routinely repeatable. The CT-related antigen is at least a useful marker of the fully virulent (i.e., fluidinducing) phenotype of $(++)$ strains. It may yet prove to be a determinant of fluid secretion.

Thus, it could be that influx of neutrophils is induced by invading organisms, and that fluid secretion arises from the interaction of these leucocytes with luminal organisms to release a toxin or an endogenous secretagogue, or both. The

\section{REFERENCES}

Clarke G J et al. 1988 Expression of an antigen in strains of Salmonella typhimurium which reacts with antibodies to cholera toxin. Journal of Medical Microbiology 25 : 139-146.

Coleman R E, Black R E, Welch D M, Maxwell J G 1980 Indium-111 labelled leukocytes in the evaluation of suspected abdominal abscesses. American Journal of Surgery 139: 99-104.

Duebbert I E, Peterson J W 1985 Enterotoxin-induced fluid accumulation during experimental salmonellosis and cholera: involvement of prostaglandin synthesis by intestinal cells. Toxicon 23: 157-172

Finkelstein R A, Marchlewicz B A, McDonald R J, BoesmanFinkelstein M 1983 Isolation and characterization of a cholera-related enterotoxin from Salmonella typhimurium. FEMS Microbiology Letters 17 : 239-241.

Giannella R A 1979 The importance of the intestinal inflammatory reaction in salmonella-mediated intestinal secretion. Infection and Immunity 23: 140-145.

Giannella R A, Formal S B, Dammin G J, Collins H 1973 Pathogenesis of Salmonellosis. Studies of fluid secretion, mucosal invasion and morphological reaction in the rabbit ileum. Journal of Clinical Investigation 52: 441-453.

Giannella R A, Gots R E, Charney A N, Greenough W B, Formal S B 1975 Pathogenesis of Salmonella mediated intestinal fluid secretion. Gastroenterology 69: 1238-1245.

Giannella R A, Rout W R, Formal S B 1977 Effect of indomethacin on intestinal water transport in salmonellainfected rhesus monkeys. Infection and Immunity 17: 136139.

Gots R E, Formal S B, Giannella R A 1974 Indomethacin inhibition of Salmonella typhimurium, Shigella flexneri and cholera-mediated rabbit ileal secretion. Journal of Infectious Diseases $130 ; 280-284$.

Hawker R J, Hall C E, Drolc Z, Rhys-Evans P H 1985 Indium bacterial phenotype is apparently more important for the genesis of fluid secretion than invasion of the intestinal mucosa: subjective examination of immunofluorescently stained sections of infected mucosa showed that old and young cultures invaded to the same degree, irrespective of fluid secretion; but these observations on invasion need to be quantified. Tests of these hypotheses have begun.

DCAC, MPO and JS gratefully acknowledge the financial support of the Wellcome Trust and the Medical Research Council.

leucocyte imaging in true pyrexia of unknown origin. European Journal of Nuclear Medicine 10: 172-174.

Higgs G A, McCall E, Youlten L J F 1975 A chemotactic role for prostaglandins released from polymorphonuclear leucocytes during phagocytosis. British Journal of Pharmacology 5: $539-546$.

Matuchansky C, Bernier J J 1973 Effect of prostaglandin $E_{1}$ on glucose, water and electrolyte absorption in human jejunum. Gastroenterology 64: 1111-1118.

Pierce N F, Carpenter C C J, Elliott H L, Greenough W B 1971 Effects of prostaglandins, theophylline and cholera exotoxin upon transmucosal water and electrolyte movement in the canine jejunum. Gastroenterology 60: 22-32.

Qi G-M, Clarke G J, Wallis T S, Stephen J 1989 The effect of cultural conditions on the expression of cholera-related antigen in Salmonella typhimurium. Journal of Medical Microbiology 30: 213-217.

Stephen J, Wallis T S, Starkey W G, Candy D C A, Osborne M $\mathrm{P}$, Haddon $\mathrm{S} 1985$ Salmonellosis: in retrospect and prospect. In: Microbial toxins and diarrhoeal disease. Ciba Foundation Symposium No. 112, Pitman, London, pp 175-192.

Wallis T S, Starkey W G, Stephen J, Haddon S J, Osborne M P Candy D C A 1986 a Enterotoxin production by Salmonella typhimurium strains of different virulence. Journal of Medical Microbiology 21 : 19-23.

Wallis T S, Starkey W G, Stephen J, Haddon S J, Osborne M P, Candy D C A $1986 b$ The nature and role of mucosal damage in relation to Salmonella typhimurium-induced fluid secretion in the rabbit ileum. Journal of Medical Microbiology 22: 39-49.

Worton K J, Candy D C A, Wallis T S, Clarke G J, Osborne M P, Haddon S J, Stephen J 1989 Studies on early association of Salmonella typhimurium with intestinal mucosa in vivo and in vitro: relationship to virulence. Journal of Medical Microbiology 29: 283-294. 